\title{
Quantum simulators, continuous-time automata, and translationally invariant systems.
}

\author{
K. G. H. Vollbrecht ${ }^{1}$ and J. I. $\operatorname{Cirac}^{1}$ \\ 1 Max-Planck Institut für Quantenoptik, Hans-Kopfermann-Str. 1, Garching, D-85748, Germany
}

(Dated: October 26, 2018)

\begin{abstract}
The general problem of finding the ground state energy of lattice Hamiltonians is known to be very hard, even for a quantum computer. We show here that this is the case even for translationally invariant systems. We also show that a quantum computer can be built in a $1 \mathrm{D}$ chain with a fixed, translationally invariant Hamitonian consisting of nearest-neighbor interactions only. The result of the computation is obtained after a prescribed time with high probability.
\end{abstract}

PACS numbers:

The difficulty of simulating the dynamics of quantum systems by classical means was recognized by Feynman 1] more than two decades ago. He proposed to use another system, a quantum simulator, to overcome this problem, introducing several visionary ideas about quantum computation. At the same time, very powerful classical techniques have been discovered, which allows us to tackle important problems in many-body physics. One of the most important questions in this context is to decide which kind of problems can (or cannot) be efficiently simulated by quantum (or classical) simulators.

While it is clear that such a device can simulate the dynamics very efficiently [2], it seems that it cannot be used to prepare ground states of arbitrary nearest neighbor interacting Hamiltonians. In fact, very recently it has been shown that if that was possible even in one spatial dimension [3], then a quantum computer would be able to solve all NP (and even QMA) problems efficiently, something which seems to be unreasonable. One may argue that systems in Nature are not so general since they typically posses certain symmetries (like homogeneity, or, equivalently, translational invariance) which restrict very much the Hamiltonians we are interested in and thus it is not so surprising that some of them cannot be efficiently simulated.

Another related question is whether a particular quantum simulator (eg. one that is translationally invariant (TI) and with nearest neighbor interactions only) may be as powerful as a general one [4]. This question can be answered in the positive if one shows that it can perform every quantum computation efficiently (with a polynomial overhead in terms of the number of qubits). In fact, we have previously shown that this is the case if one is able to change the evolution Hamiltonian with time [5, 6]. Very recently, Werner et al. [7] have also shown that it is possible by alternating two kind of discrete gates, a result which generalizes previous ones on cellular automata. However, it still remains to be seen if with a fixed Hamiltonian that is TI and which only includes nearest neighbor interactions it is still possible to perform arbitrary quantum computations efficiently.

In this paper we address the two problems mentioned above. First, we show that by demanding that a $1 \mathrm{D}$
Hamiltonian is TI, a quantum computer is not more efficient in preparing its ground state. Note that in order to be able to accommodate the number of parameters which define QMA problems, we must relax the condition of only nearest-neighbor interactions, although we still keep only three-body (sites) interactions. Thus, our results imply that the homogenity that typically appears in Nature is not enough to make it simulatable. Second, we show that it is possible to build a quantum computer based on a static, TI Hamiltonian with only nearestneighbor interactions, as long as one is able to prepare arbitrary product states and measure the sites independently. This result extends previous ones on cellular automata, but as opposed to those, has no simple classical analogue since we deal here with continuous time evolution. The TI quantum computation scheme can be seen as a combination of a cellular automata approach with a continuous-time quantum walk [1, 7].

Ground state energy in TI systems: We start out by showing that finding the ground state energy $E_{0}$ of any TI Hamiltonian in a $1 \mathrm{D}$ chain is very difficult (as we increase the number of sites), even for a quantum computer or simulator. In fact, we show that for each QMAcomplete problem one can find a TI Hamiltonian, $H_{n}$, such that: $E_{0}=0$ if the answer to the problem is 'yes'; $E_{0}>1 /(\operatorname{Poly}(n))$, if the answer is 'no'. Here $\operatorname{Poly}(n)$ denotes any polynomial in $n$. This implies that any effective routine for finding the ground state energy would give us a possibility to efficiently solve all problems in the complexity class QMA. Our result heavily relies on the recent discovery [3] that QMA-complete problems can be encoded in the ground state energy of a Hamiltonian $h_{n}$ describing a 1D chain of $n$ system with $d=12$ levels each and with nearest-neighbor interactions, such that its minimum eigenvalue, $\lambda_{\min }$, is either zero or $\lambda_{\min }>\operatorname{Poly}(n)$. Our strategy is to build $H_{n}$ out of $h_{n}$ by only increasing the dimension $d$ by a factor of two.

We take at every site one additional qubit and define the Hamiltonian $H_{n}^{i}=|1\rangle\left\langle\left. 1\right|_{i} \otimes h_{n}^{(i, \ldots, i+n)}\right.$. That is, if the extra qubit at site $i$ is in the state $|1\rangle$, then the Hamiltonian $h_{n}$ is applied to the $(i, \ldots, i+n)$ particles in the chain in this order, where we identify the sites separated 
by $n+1$ sites. Furthermore, we define the Hamiltonian $H^{\prime}$ that only acts one the extra qubits as

$H^{\prime}=\frac{1}{n(n-1)}\left[1-\sum_{k}\left|1_{k}\right\rangle\left\langle 1_{k}\left|+\sum_{k^{\prime \prime} \neq k^{\prime}}\right| 1_{k^{\prime}} 1_{k^{\prime \prime}}\right\rangle\left\langle 1_{k^{\prime}} 1_{k^{\prime \prime}}\right|\right]$,

where $k, k^{\prime}, k^{\prime \prime}$ run from site 1 to $n$. Now, we take

$$
H_{n}=H^{\prime}+\frac{1}{n} \sum_{i=1}^{n} H_{n}^{i}
$$

This Hamiltonian is translationally invariant, and contains up to three-body interactions only. It is easy to show that it fulfills the conditions mentioned above, since its eigenvectors have the form $\left|k_{1}, k_{2}, \ldots\right\rangle \otimes|\phi\rangle$, where $\left|k_{1}, k_{2}, \ldots\right\rangle$ is a product state on the extra qubits only and $|\phi\rangle$ is a state on the rest of the chain. In case $h_{n}$ has a zero eigenvalue, with corresponding eigenvector $\left|\phi_{0}\right\rangle$, we can construct an eigenvector of $H_{n},|1,0,0, \ldots, 0\rangle \otimes\left|\phi_{0}\right\rangle$, also with $E_{0}=0$. Otherwise, it is easy to show that the minimal eigenvalue of $H_{n}$ is lower bounded by $\min \left(\frac{\lambda_{\min }}{n}, \frac{1}{n^{2}(n-1)}\right)$, given that if there is no or more than one extra qubit in 1 , this is penalized in energy by $H^{\prime}$, whereas if there is only one, then this is penalized by $H_{n}^{i}$.

Continuous-time automata: Now, we introduce a programmable quantum computation scheme for an infinite chain of quantum systems of dimension $d=30$. The program is encoded into the initial state, while the time evolution is fixed and given by a universal TI Hamiltonian with nearest-neighbor interaction only. We will start by introducing a simple standard quantum computing scheme. Later on, we will show how this quantum computer can be simulated by a continuous time evolution.

We consider a simple quantum computer with an $n-$ qubit 'hard disk' and a read/write head, that we call the pointer. This pointer can be moved such that we can address single qubits. Furthermore, the pointer has an internal quantum state, a qubit. To perform any quantum computation we write a program consisting of five different commands: L) The pointer is moved one site to the left; R) the pointer is moved one site to the right; $\mathrm{S})$ the qubit at the position of the pointer and the internal state of the pointer are swaped; G) a G-Gate [7] on those two qubits is applied. The G-gate allows for arbitrary quantum computations if it can be applied between any two qubits. In this simple model this can be accomplished by loading qubits with the S-command into the internal pointer state, which can then be moved to any other qubit. We now encode this quantum computer into a higher dimensional chain in the following way: Every site in the chain has three registers. A 'qubit' register having dimension 2. It acts like the normal qubit of the quantum computer. A 'pointer' register having dimension 3 . Here we encode the pointer, where 0 indicates no 'program‘

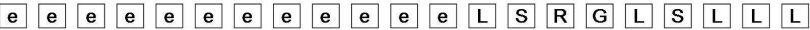

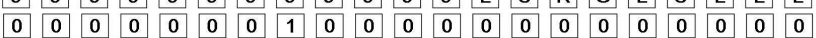

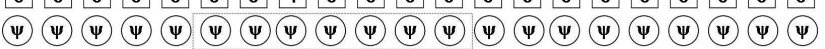
'Quantum Computer'

FIG. 1: A 7-qubit quantum computer encoded into a one dimensional chain. The pointer is represented by the 1 , the program is encoded right of the quantum computer.

pointer and $1 / 2$ the presence of a pointer with an internal qubit state. Finally, a 'program' register having dimension 5: One for 'e=empty' and the rest for commands $\{L, R, S, G\}$. The total dimension of one site defined this way matches 30 .

We choose $n$ neighboring sites to be the 'quantum computer', i.e., the qubit-registers of those sites correspond one to one to the qubits of the quantum-computer we want to simulate (see Fig. 1). The pointer registers are in state $|0\rangle$ everywhere, except for one site which contains $|1\rangle$. The program is written in the program register, an area to the right of the 'quantum computer', where the commands are arranged in the order they should be executed from left to right. The rest of the program registers are filled with $|e\rangle$.

We assume now a TI Hamiltonian with nearest neighbor interactions, $H=\sum_{i} H_{i}$, where $H_{i}$ denotes a two-site Hamiltonian acting only on the sites $i$ and $i+1$ and is given by

$$
H_{i}=\sum_{C \in\{L, R, S, G\}}|e\rangle_{i}\langle C|\otimes| C\rangle_{i+1}\langle e| \otimes U_{i, i+1}^{C}+\text { h.c. }
$$

The first two operators only act on the two program registers at sites $i$, and $i+1$, respectively, while $U^{C}$ 's are unitary operators acting both on the qubit and pointer register at those two sites. The unitaries $U^{S}$ and $U^{G}$ act only on the first site $i$. A swap resp. G-gate is applied to both the $i$-th qubit and the internal state of the pointer in case a pointer is present, and the identity operation otherwise. $U^{R}$ swaps the two pointer sites while $U^{L}$ swaps the two qubit sites. Let us assume now, that we apply one of those unitaries on every pair of sites $(i, i+1)$ starting from the right end of the chain going step by step to the left. $U^{S}$ and $U^{G}$ will do nothing until we reach the position where a pointer is present in the first of the two participating sites. In this case, the corresponding gates are applied. The $U^{R}$ gate will swap the two pointer states at every position. As a result the pointer, marked by $|1\rangle$, is moved one step to the right. Finally, $U^{L}$ will swap the two qubit registers. As a result the whole qubit-register chain is moved one step to the right. Relative to the 'quantum computer' position, the pointer is moved one step to the left. Therefore, if such a sequence of unitaries is applied from right to left the corresponding set of commands is applied to the 'quantum computer'. Note, that it is sufficient to start this 
sequence of unitaries at any position to the right of the 'quantum commuter' and to stop it once they are to its left. Our claim is now that this is exactly what the time evolution basically does.

To this aim, we consider the time evolution operator $e^{-i H t}=\sum_{n} \frac{(-i H t)^{n}}{n !}$. Applied to the initial state $\mid$ start $\rangle$ we just end up in a linear combination of states of the form $H^{n} \mid$ start $\rangle$. Since $H$ is defined as a sum of terms of the form $h_{i}^{C}:=\left|e_{i}, C_{i+1}\right\rangle\left\langle C_{i}, e_{i+1}\right| \otimes U_{i, i+1}^{C}$ (and the hermitian conjugates), we can write the result of a time evolution as a linear combination of states of the form $h_{n} \ldots h_{3} h_{2} h_{1} \mid$ start $\rangle$, where every $h_{k} \in\left\{h_{i}^{C}, h_{i}^{C^{\dagger}}\right\}$. The effect of any such $h_{i}^{C}$ on a state is the following: it either moves the command $C$ from place $(i+1)$ to $i$ and applies $U^{C}$ to both sites $(i, i+1)$, or it maps the state to zero if no $C$ is at $i+1$ and no empty space $|e\rangle$ at $i$. In the same manner $h_{i}^{C \dagger}$ results in a state where the command $C$ is moved from $i$ to $i+1$ while $U^{C^{\dagger}}$ is applied. Therefore $h_{n} \ldots h_{3} h_{2} h_{1} \mid$ start $\rangle$ is either zero, or a state where several of the commands have moved while applying the assigned unitaries (or their conjugates) all the way from their initial to their final position. Note that such a command can only move, if the corresponding neighboring site is in the state $|e\rangle$. This implies that, up to some $|e\rangle$ 's in between, the order of the commands in the program register can never change. Further note that if a command moves to the left and afterwards to the right, we end up in the same state since $U^{C}$ and $U^{C^{\dagger}}$ cancel each other. This implies that two states $h_{n} \ldots h_{3} h_{2} h_{1} \mid$ start $\rangle, g_{m} \ldots g_{3} g_{2} g_{1} \mid$ start $\rangle$ are equal, iff the configuration of commands in the program registers are equal.

Assume now that we measure the program registers in the standard product basis after some time evolution. According to the above discussion we can conclude just from the configuration of the program register what has happened to the pointer and qubit registers. In particular, suppose that we measure a configuration where all the commands that were initially to the right of the 'quantum computer'(see Fig. 1), are found to the left of the 'quantum computer'. In this case the whole program has been executed (in the right order) to the 'quantum computer' and we can read out the result. If this is not the case, we can in principle continue time evolution and repeat until we found a positive result. To boost the probability of success, we can increase the program code by some irrelevant code, e.g. by adding $|L\rangle^{\prime} s$ that do not effect the result of the computation if carried out after the real program. This irrelevant code will act as a kind of barrier, that prevents the real program code to move to the right and forces it to do the computation.

In order to verify that this is an effective way of carrying out quantum computations, we have to guarantee that the probability of finding the whole program to the left of the 'quantum computer' is finite after a finite time (that only grows polynomially in the number of qubits). To this end we have to solve the above problem and calculate (a lower bound to) the probability of success. Note, that given the initial state, the whole system stays in a subspace that can be labelled by only the configuration of the commands in the program register. So, we can map our system onto a chain of qubits, where $|0\rangle$ means 'empty' and $|1\rangle$ means 'command'. Note that, since the order of the commands stays unchanged under the evolution, we do not have to distinguish between the different commands. The Hamiltonian simplifies to $H=\sum_{i} H_{i}$ with $H_{i}=\left|1_{i} 0_{i+1}\right\rangle\left\langle 0_{i} 1_{i+1}\right|+h . c$. . At this point we see that the efficiency of the computation does not depend on the specific program we want to run. Let us take this system as electrons in a lattice, where $|1\rangle$ stands for an 'electron' and $|0\rangle$ for an empty site and $H_{i}$ is a hopping term, allowing the electron to hop from one site to a neighboring site. As usual for fermions, there are no hopping terms allowed resulting in two electrons sitting at the same site. Thus, we end up with a system of non-interacting fermions in second quantization. To solve it, it is simple to go back into first quantization, where we just have to consider a single electron Hamiltonian, $H=\sum_{k}|k+1\rangle\langle k|+h . c$. , and all the effects coming from the Pauli principle are automatically implied by the anti-symmetrization. The single electron problem can be easily solved. Let us assume $M$ sites with periodic boundary conditions. The eigenvectors are given by $\psi_{q}=\frac{1}{\sqrt{M}} \sum_{x} e^{i \frac{2 p i}{M} x q}|x\rangle$ with corresponding eigenvalues $\varepsilon(q)=2 \cos \left(\frac{2 \pi}{M} q\right)$.

Let us now look at $M$ sites where the first $N$ sites are filled with each one electron. Remember, that these $N$ electrons correspond to the program and enough irrelevant code to force the program to go into the desired direction. An electron sitting at site $y$ is written in terms of the eigenvectors as $\phi_{y}=\frac{1}{M} \sum_{q} \psi_{q} e^{-i \frac{2 \pi}{M} y q}=$ $\frac{1}{M} \sum_{q, k} e^{i \frac{2 \pi}{M}(x-y) q}|x\rangle$. After the time evolution, the state is changed into $\phi_{y, t}=\frac{1}{M} \sum_{q, k} e^{i \frac{2 \pi}{M}(x-y) q+i \varepsilon(q) t}|x\rangle$. We assume now $N$ electrons sitting in the first $N$ sites what leads to the state $\psi_{0}=S\left[\left|\phi_{1}, \phi_{2}, \phi_{3}, \ldots, \phi_{N}\right\rangle\right]$, where $S$ denotes the fermionic anti-symmetrization operator. After waiting a time $t$ we end up in the state $\psi_{t}=S\left[\left|\phi_{1, t}, \phi_{2, t}, \phi_{3, t}, \ldots, \phi_{N, t}\right\rangle\right]$. We now want to compute the probability $p_{1}$ to find particle 1 at time $t$ still in one of the first $N$ sites. Note that, since the vectors $\phi_{y, t}$ are still orthogonal, the reduction to the first particle gives us an averaging over all the starting positions $y$. This probability yields

$$
p_{1}=\frac{1}{N} \sum_{y=1}^{N} \sum_{x=1}^{N}\left|\left\langle\phi_{y, t} \mid x\right\rangle\right|^{2} .
$$

In the Appendix it is shown that by choosing $t$ to be proportional to $N$, e.g. $t=5000 N$, we can bound this probability to be smaller than a fixed constant, e.g. $p_{1}<0.3$. 
So we can guarantee to find particle 1 after a polynomial time with probability bigger than $p=0.7$ outside the starting area. From this we can calculate the expected number of electrons that will be found in those areas. They will be $N p_{1}$ and $N\left(1-p_{1}\right)=N p$. For a successful computation a fixed number $k$ of electrons have to leave their starting area. But what is the probability to find more than $k$ electrons outside the starting area if we know that the expectation value is $N p$ ? Let us assume the worst case scenario: We either get $(k-1)$ electrons with probability $p_{f}$ or $N$ electrons with probability $p_{s}$. Since we know that $p_{f}(k-1)+p_{s} N=N p$ we can conclude that the success probability $p_{s}>\frac{1-k+N p}{1-k+N}$ which can be made arbitrarily close to $p$ by choosing $N$ to grow polynomially with $k$. Now let us apply this to our model. Note that, due to the symmetry of the problem, the number of electrons that moved to the left of the starting position and to the right of the starting position will be the same. We will therefore assume all electrons moving in the right direction, what can be corrected by an irrelevant factor of two for all length in the following discussion. We have a program of length $l_{p}$ and a quantum computer of length $l_{q}$. Instead of of searching for $l_{p}$ 'electrons' to the left of the 'quantum computer' we can search for $l_{p}+l_{q}$ 'electrons' leaving the starting area. The extra $l_{q}$ 'electrons' will be just part of the irrelevant code; that guarantees that the real program completely passed the quantum computer. Then we choose $N$ to be e.g. $\left(l_{p}+l_{q}\right)^{2}$. The above calculation then tells us that after a time of $5000\left(l_{p}+l_{q}\right)^{2}$ the computation is successful with a probability higher than $p=0.7$ (if we do not assume the worst case it is quite likely that we approach 1 ). Since $l_{p}$ and $l_{q}$ grows only polynomial the same holds for the evolution time.

Conclusion: We have shown that calculating the ground state energy of a translationally invariant Hamiltonians in 1D is as hard as solving QMA-problems. We have also introduced a programable quantum computation scheme using one fixed translationally invariant nearest-neighbor Hamiltonian. The program is encoded in the initial state. The computation itself requires only enough patience but no active further control. We acknowledges support from EU projects (SCALA and CONQUEST), DFG-Forschungsgruppe 635, and the excellence clusters MAP and NIM. K.V. thanks Frank Verstraete for helpful discussions.

Appendix: For equation (2) we get

$$
p_{1}=\frac{1}{N M^{2}} \sum_{x, y=1}^{N} \sum_{q, q^{\prime}=1}^{M} \cos \left[\left(q-q^{\prime}\right)(x-y) \frac{2 \pi}{M}+\Delta t\right]
$$

where $\Delta=\varepsilon(q)-\varepsilon\left(q^{\prime}\right)$. We assume that $N$ and $M$ are large and $M \gg N$. If we sum over the cases $q=q^{\prime}$ we get $N / M$. Since this term vanishes in the limit of $M \gg N$, we can ignore those cases. Let us now approximate the sums over $x$ and $y$ by integrals

$$
\frac{N}{M^{2}} \sum_{q, q^{\prime}=1}^{M} \int_{X, Y=0}^{1} d X d Y \cos \left[\left(q-q^{\prime}\right)(X-Y) N \frac{2 \pi}{M}+\Delta t\right](4)
$$

These integrations leads to

$$
\frac{1}{N \pi^{2}} \sum_{q, q^{\prime}=1}^{M} \frac{\cos (\Delta t)\left[\sin \left(\frac{N}{M}\left(q-q^{\prime}\right) \pi\right)\right]^{2}}{\left(q-q^{\prime}\right)^{2}} .
$$

Now let us define $\delta=\left|q-q^{\prime}\right|$. Note that all terms only depend on the absolute value $q-q^{\prime}$ and that we already neglected the terms with $q-q^{\prime}=0$. We get $p_{1}=\frac{1}{N \pi^{2}} \sum_{\delta=1}^{M} g(\delta) f(\delta)$. with $g(\delta)=\frac{\left[\sin \left(\frac{N}{M} \delta \pi\right)\right]^{2}}{\delta^{2}}$ and $f(\delta)=2 \sum_{q=1}^{M-q} \cos (\varepsilon(q)-\varepsilon(q+\delta)) t$. Note that for $t=0$ we get that $f(\delta)=2(M-q)$ and in this case the overall sum converges to 1 . One observation is that the main contribution to the sum comes from the parts where $\delta$ is smaller than $2 M / N$. Furthermore for $t>0$ we get $f(\delta) \leq 2(M-q)$. To get a bound we now want to calculate $f(\delta)$ in the relevant region $\delta<2 M / N$. Since $\delta \frac{2 \pi}{M}$ is small in this region, we can approximate $\varepsilon(q)-\varepsilon(q+\delta)$ by the derivative $\varepsilon^{\prime}(q) \frac{2 \pi}{M}$ leading to $f(\delta)=2 \sum_{q=1}^{M-q} \cos \left[\sin \left(\frac{2 \pi}{M} q\right) 2 t \delta 2 \pi / M\right]$. This can be solved by converting the sum into an integral leading to $f(\delta)=2 M \operatorname{JBessel}(0,4 t \delta \pi / M))[8]$. To get an upper bound we split up the sum in three terms

$p_{1}=\sum_{\delta=1}^{\varepsilon M / N} g(\delta) f(\delta)+\sum_{\delta=\varepsilon M / N}^{2 M / N} g(\delta) f(\delta)+\sum_{\delta=2 M / N}^{M} g(\delta) f(\delta)$

Assuming $f(\delta)=2 M$ for the first term we get for small $\varepsilon$ a bound of $2 \varepsilon$. The second term we get as bound $\max [f(q) / 2 M, \varepsilon M / N<q<2 M / N]$. The last term we can numerical calculate for large $M$ and get it to be smaller then 0.05 . If we set in $\varepsilon=0.001, t=5000 N$ we get that $p_{1} \leq 0.3$.

[1] R. Feynman, International Journal of Theoretical Physics 21, 467 (1982); R. Feynman, Found. Phys., 16(6):50731, 1986.

[2] S. Lloyd, Science 273, 1073 (1996); C. Zalka, Proc. Roy. Soc. Lond. A 454, 313 (1998).

[3] D. Gottesman et al. (private communication); A. Yu. Kitaev, A. H. Shen, M. N. Vialyi, Classical and Quantum Computation, Americal Mathematical Society (2002); J. Kempe, A. Kitaev, O. Regev, SIAM Journal of Computing, Vol. 35(5),1070 (2006); R. Oliveira, B. Terhal, quant-ph/0504050

[4] Christina V. Kraus, Michael M. Wolf, and J. Ignacio Cirac, arXiv:quant-ph/0607094

[5] K. G. H. Vollbrecht, E. Solano, and J. I. Cirac, Phys. Rev. Lett. 93, 220502 (2004). 
[6] K. G. H. Vollbrecht, and J. I. Cirac, Phys. Rev. A 73, 012324 (2006)

[7] D.J. Shepherd, T. Franz, R.F. Werner, Phys. Rev. Lett. 97 (2006) 020502; Diego de Falco, Dario Tamascelli, J. Phys.
A: Math. Gen. 39 5873-5895 (2006)

[8] Mathematica 5.0.0.0., WolframResearch (2003). 